\title{
Lewis-Riesenfeld invariants and transitionless quantum driving
}

\author{
Xi Chen, ${ }^{1,2}$ E. Torrontegui, ${ }^{1}$ and J. G. Muga ${ }^{1}$ \\ ${ }^{1}$ Departamento de Química-Física, UPV-EHU, Apdo 644, E-48080 Bilbao, Spain, \\ ${ }^{2}$ Department of Physics, Shanghai University, 200444 Shanghai, China \\ (Received 16 February 2011; published 22 June 2011)
}

\begin{abstract}
Different methods have been recently put forward and implemented experimentally to inverse engineer the time-dependent Hamiltonian of a quantum system and accelerate slow adiabatic processes via nonadiabatic shortcuts. In the "transitionless quantum driving" proposed by Berry, shortcut Hamiltonians are designed so that the system follows exactly, in an arbitrarily short time, the approximate adiabatic path defined by a reference Hamiltonian. A different approach is based on first designing a Lewis-Riesenfeld invariant to carry the eigenstates of a Hamiltonian from specified initial to final configurations, again in an arbitrary time, and then constructing from the invariant the transient Hamiltonian that connects these boundary configurations. We show that the two approaches, apparently quite different in form and so far in results, are, in fact, strongly related and potentially equivalent, so that the inverse-engineering operations in one of them can be reinterpreted and understood in terms of the concepts and operations of the other one. We study, as explicit examples, expansions of time-dependent harmonic traps and the state preparation of two-level systems.
\end{abstract}

DOI: 10.1103/PhysRevA.83.062116

PACS number(s): 03.65.Xp, 37.10.De, 32.80.Qk, 03.65.Ca

\section{INTRODUCTION}

Externally imposed time-dependent interactions are frequently varied slowly to keep adiabaticity and control the final state of a quantum system robustly versus parameter fluctuations. There are, however, many instances where we would like or need to quicken these operations. If they take too long, they will be impractical for applications with many repetitions, e.g., to manipulate quantum information by transporting ions or atoms [1], or they may suffer from decoherence, noise, or losses, so speeding them up may be the only way to actually implement the ideal final outcome. Moreover, in many experiments, as in atomic fountain clocks, high repetition rates contribute to the achievement of better signal-to-noise ratios and better accuracy [2]. Adiabatic steps are also the bottleneck in some cyclic processes. They determine, for example, the cooling rates in quantum refrigerators and quantify the unattainability of absolute zero [3-5].

Recently, several works have been devoted to theoretical proposals [3,6-20] or experimental realizations [21-25] of fast nonadiabatic shortcuts to the states reached by slow adiabatic processes in matter wave expansions or compressions, splitting, and transport. Berry, in particular, has proposed a "transitionless quantum driving" (also known as "transitionless tracking algorithm") to design time-dependent interactions so that the system follows exactly, in an arbitrarily short time, the approximate adiabatic path defined by a reference, zeroth order Hamiltonian [10]. This method has been applied to speed up adiabatic passage techniques and achieve fast and robust population control in two- and three-level atomic systems [12]. A different approach is based on first designing a Lewis-Riesenfeld invariant [26] to carry the eigenstates of a Hamiltonian from initial to final configurations, again in an arbitrary time, and then constructing from the invariant a transient, driving Hamiltonian [8,9]. These methods were compared for harmonic oscillator expansions for which they provided rather different shortcut paths [15].

Berry mentioned the existence of connections between the invariants and the transitionless algorithm for a two-state system without pursuing them further [10]. Following that hint, we show in this work that these two approaches are in fact closely related, and can be stated in common terms, so that the inverse-engineering operations in one of them can be reinterpreted using concepts and operations of the other one. This sets their potential equivalence. The different results that have been found so far, as explained in detail below, are due to the ample freedom offered by both approaches to construct the driving shortcut Hamiltonian. We study the general setting as well as two explicit examples: expansions of time-dependent harmonic traps and the state preparation in two-level systems.

A word of caution on notation: as we shall deal with different methods and examples, multiple usage of some symbols, such as $\left|\phi_{n}\right\rangle,|n\rangle, H, I, \lambda_{n}, U$, and $E_{n}$, is unavoidable unless we load them with subscripts and superscripts; so consistency is strictly guaranteed only within each section. In most cases, this repeated usage will suggest a possible relation. The context and explanations will clarify how this comes about.

\section{GENERAL FRAMEWORK}

\section{A. Lewis-Riesenfeld invariants}

We shall describe first Lewis-Riesenfeld theory in a nutshell [26]. Let us consider a quantum system evolving with a time-dependent Hamiltonian $H(t)$. A dynamical invariant $I(t)$ satisfies

$$
i \hbar \frac{\partial I(t)}{\partial t}-[H(t), I(t)]=0,
$$

so that its expectation values remain constant in time. $I(t)$ can be used to express an arbitrary solution of the time-dependent Schrödinger equation

$$
i \hbar \frac{\partial}{\partial t}|\Psi(t)\rangle=H(t)|\Psi(t)\rangle
$$

as a superposition of "dynamical modes" $\left|\psi_{n}(t)\right\rangle$ :

$$
|\Psi(t)\rangle=\sum_{n} c_{n}\left|\psi_{n}(t)\right\rangle,
$$




$$
\left|\psi_{n}(t)\right\rangle=e^{i \alpha_{n}(t)}\left|\phi_{n}(t)\right\rangle
$$

where $n=0,1, \ldots, c_{n}$ are time-independent amplitudes, $\left|\phi_{n}(t)\right\rangle$ are orthonormal eigenvectors of the invariant $I(t)$, a Hermitian operator satisfying $I(t)\left|\phi_{n}(t)\right\rangle=\lambda_{n}\left|\phi_{n}(t)\right\rangle$, with $\lambda_{n}$ real constants, and the Lewis-Riesenfeld phases are defined as [26]

$$
\alpha_{n}(t)=\frac{1}{\hbar} \int_{0}^{t}\left\langle\phi_{n}\left(t^{\prime}\right)\left|i \hbar \frac{\partial}{\partial t^{\prime}}-H\left(t^{\prime}\right)\right| \phi_{n}\left(t^{\prime}\right)\right\rangle d t^{\prime} .
$$

We use, for simplicity, a notation for a discrete spectrum of $I(t)$, but the generalization to a continuum or mixed spectrum is straightforward. We also assume a nondegenerate spectrum.

\section{B. Invariant based inverse engineering}

Suppose that we want to drive the system from an initial Hamiltonian $H(0)$ to a final one $H\left(t_{f}\right)$, such that the populations in the initial and final instantaneous bases are the same but admitting transitions at intermediate times. To inverse engineer a time-dependent Hamiltonian $H(t)$ and achieve this goal, we may define first the invariant through its eigenvalues and eigenvectors as

$$
I(t)=\sum_{n}\left|\phi_{n}(t)\right\rangle \lambda_{n}\left\langle\phi_{n}(t)\right| .
$$

The Lewis-Riesenfeld phases may also be chosen as arbitrary functions to write down the time-dependent unitary evolution operator $U$ [see Eqs. (3) and (4)] as

$$
U=\sum_{n} e^{i \alpha_{n}(t)}\left|\phi_{n}(t)\right\rangle\left\langle\phi_{n}(0)\right| .
$$

It must obey

$$
i \hbar \frac{\partial}{\partial t} U=H(t) U
$$

which we solve formally for the Hamiltonian,

$$
H(t)=i \hbar\left(\partial_{t} U\right) U^{\dagger},
$$

that is,

$$
H(t)=F(t)+i \hbar \sum_{n}\left|\partial_{t} \phi_{n}(t)\right\rangle\left\langle\phi_{n}(t)\right|,
$$

where $F(t)$ is diagonal in the basis of the invariant:

$$
F(t)=-\hbar \sum_{n}\left|\phi_{n}(t)\right\rangle \dot{\alpha}_{n}\left\langle\phi_{n}(t)\right|
$$

and the dot denotes the derivative with respect to time. Note that for a given invariant, there are many possible Hamiltonians corresponding to different choices of phase functions $\alpha_{n}(t) .{ }^{1}$ In general, $I(0)$ does not commute with $H(0)$, which means that the eigenstates of $I(0),\left|\phi_{n}(0)\right\rangle$, do not coincide with the eigenestates of $H(0) . H\left(t_{f}\right)$ does not necessarily commute with $I\left(t_{f}\right)$ either. If we impose $[I(0), H(0)]=0$ and $\left[I\left(t_{f}\right), H\left(t_{f}\right)\right]=0$, the eigenstates coincide and then a state transfer without final excitations is guaranteed. In a typical

\footnotetext{
${ }^{1}$ To connect with Lohe's work [27], we may also write $I(t)=$ $T I(0) T^{\dagger}$ in terms of the unitary operator $T=\sum_{n}\left|\phi_{n}(t)\right\rangle\left\langle\phi_{n}(0)\right|$.
}

application, the Hamiltonians $H(0)$ and $H\left(t_{f}\right)$ are given; they set the initial and final boundaries for the process, and we use these boundary conditions to define $I(t)$ and its eigenvectors accordingly. A convenient, although by no means necessary, relation is to set $I(0)=H(0)$.

While the $\alpha_{n}(t)$ may be taken as fully free time-dependent phases in some applications, the choice of $\alpha_{n}(t)$ may also be constrained by a preimposed or assumed structure of $H(t)$. $H(t)$ may, for example, be set initially as a given function of parameters whose time dependence is not yet specified, as in the ordinary harmonic oscillator with a frequency whose time dependence is not yet fixed initially. If the assumed structure of $H(t)$ leads to a specific structure of $I(t)$ and the $\alpha_{n}$, the inverse method can be applied so that $I(t)$ and $\alpha_{n}(t)$ are fully specified first within the forms that guarantee the imposed structure of $H(t)$. We shall see examples of how this works in Secs. III and IV.

\section{Transitionless tracking algorithm}

In Berry's method [10], the starting point is a timedependent reference Hamiltonian

$$
H_{0}(t)=\sum_{n}\left|n_{0}(t)\right\rangle E_{n}^{(0)}(t)\left\langle n_{0}(t)\right|
$$

for which the approximate time-dependent adiabatic solutions are

$$
\left|\psi_{n}(t)\right\rangle=e^{i \xi_{n}(t)}\left|n_{0}(t)\right\rangle
$$

where the adiabatic phases, with dynamical and geometric parts, are

$$
\xi_{n}(t)=-\frac{1}{\hbar} \int_{0}^{t} d t^{\prime} E_{n}^{(0)}\left(t^{\prime}\right)+i \int_{0}^{t} d t^{\prime}\left\langle n_{0}\left(t^{\prime}\right) \mid \partial_{t^{\prime}} n_{0}\left(t^{\prime}\right)\right\rangle .
$$

The approximate adiabatic vectors in Eq. (13) are defined differently from the dynamical modes of Eq. (4), but they may potentially coincide, as discussed below, so, with some caution, we use the same notation. Defining now the unitary operator

$$
U=\sum_{n} e^{i \xi_{n}(t)}\left|n_{0}(t)\right\rangle\left\langle n_{0}(0)\right|,
$$

a Hamiltonian $H(t)$ can be constructed, using again Eq. (9), to drive the system exactly along the adiabatic paths of $H_{0}(t)$ as

$$
\begin{aligned}
H(t)= & H_{0}(t)+H_{1}(t), \\
H_{1}(t)= & i \hbar \sum_{n}\left[\left|\partial_{t} n_{0}(t)\right\rangle\left\langle n_{0}(t)\right|\right. \\
& \left.-\left\langle n_{0}(t) \mid \partial_{t} n_{0}(t)\right\rangle\left|n_{0}(t)\right\rangle\left\langle n_{0}(t)\right|\right],
\end{aligned}
$$

where $H_{1}(t)$ is purely nondiagonal in the $\left\{\left|n_{0}(t)\right\rangle\right\}$ basis.

We may change the functions $E_{n}^{(0)}(t)$, responsible for the dynamical part of the phase, and, therefore, $H_{0}(t)$ itself, keeping the same $\left|n_{0}(t)\right\rangle$ eigenvectors. We could, for example, make all the $E_{n}^{(0)}(t)$ zero to suppress the dynamical phases, or compensate the geometric phase to have $\xi_{n}(t)=0$ [10]. Therefore, the Hamiltonian can be generally written in terms of the phases as

$$
H(t)=G(t)+i \hbar \sum_{n}\left|\partial_{t} n_{0}(t)\right\rangle\left\langle n_{0}(t)\right|,
$$


where

$$
G(t)=-\hbar \sum_{n}\left|n_{0}(t)\right\rangle \dot{\xi}_{n}\left\langle n_{0}(t)\right|
$$

is diagonal in the instantaneous basis of $H_{0}(t)$. Subtracting $H_{1}(t), H_{0}(t)$ may also be written as

$$
H_{0}(t)=\sum_{n}\left|n_{0}(t)\right\rangle\left[i \hbar\left\langle n_{0}(t) \mid \partial_{t} n_{0}(t)\right\rangle-\hbar \dot{\xi}_{n}\right]\left\langle n_{0}(t)\right| .
$$

In general, it is required that $H_{1}(t)$ vanish for $t<0$ and $t>t_{f}$, either suddenly or continuously at the extreme times. In that case, the $\left|n_{0}(t)\right\rangle$ become, also at the extreme times (at least at $t=0^{-}$and $t=t_{f}^{+}$), eigenstates of the full Hamiltonian.

Using Eq. (1) and the orthonormality of the $\left\{\left|n_{0}(0)\right\rangle\right\}$, we may write invariants of $H(t)$ with the form

$$
I(t)=\sum_{n}\left|n_{0}(t)\right\rangle \lambda_{n}\left\langle n_{0}(t)\right|,
$$

where the $\lambda_{n}$ are constant eigenvalues of $I(t)$. For the simple choice $\lambda_{n}=E_{n}^{(0)}(0)$, then $I(0)=H_{0}(0)$.

Up to now, we have presented the invariant-based and tracking algorithm approaches in a common manner to make their relations obvious. By reinterpreting the phases of Berry's method as $\xi_{n}(t)=\alpha_{n}(t)$, and the states as $\left|n_{0}(t)\right\rangle=\left|\phi_{n}(t)\right\rangle$, we may immediately equate $G(t)=F(t)$ and the Hamiltonians $H(t)$ in Eqs. (10) and (17). We may also find the $H_{0}(t)$ implicit in the invariant's method using Eq. (19). In other words, the dynamical modes in the invariant-based method can be also understood as approximate adiabatic modes of a certain Hamiltonian $H_{0}(t)$.

An important caveat is that, although the two methods could coincide, they do not have to. Given $H(0)$ and $H\left(t_{f}\right)$, there is much freedom to connect them using different invariants, phase functions, and reference Hamiltonians $H_{0}(t)$. It should be clear by now that each of these methods does not provide just a unique shortcut but entire families of them, a welcome flexibility that allows us to optimize the path according to physical criteria and/or operational constraints [16].

In the following sections, we shall work out two specific examples where the connections, differences, and similarities of the two approaches are illustrated and examined further.

\section{TIME-DEPENDENT HARMONIC OSCILLATOR}

\section{A. Lewis-Riesenfeld invariants}

The Schrödinger picture Hamiltonian of a particle in a harmonic oscillator with mass $m$ and time-dependent angular frequency (all "frequencies" hereafter are angular frequencies) $\omega(t)$ is

$$
H(t)=\frac{1}{2 m} \hat{p}^{2}+\frac{m \omega^{2}(t)}{2} \hat{q}^{2}
$$

where $\hat{q}$ and $\hat{p}$ are canonical conjugate position and momentum operators. The instantaneous eigenstates, in coordinate representation, and energies are, respectively,

$$
\begin{aligned}
\langle q \mid n(t)\rangle= & \left(\frac{m \omega(t)}{\pi \hbar}\right)^{1 / 4} \frac{1}{\left(2^{n} n !\right)^{1 / 2}} \\
& \times \exp \left[-\frac{m \omega(t)}{2 \hbar} q^{2}\right] H_{n}\left(\sqrt{\frac{m \omega(t)}{\hbar}} q\right),
\end{aligned}
$$

where $H_{n}$ is a Hermite polynomial, and $E_{n}(t)=(n+$ $1 / 2) \hbar \omega(t)$, so that $H(t)|n(t)\rangle=E_{n}(t)|n(t)\rangle$. The invariant form is found by trying a quadratic ansatz [26-29]:

$$
I(t)=\frac{1}{2}\left[\left(1 / b^{2}\right) \hat{q}^{2} m \omega_{0}^{2}+\frac{1}{m} \hat{\pi}^{2}\right],
$$

where $\hat{\pi}=b(t) \hat{p}-m \dot{b} \hat{q}$ plays the role of a momentum conjugate to $\hat{q} / b$. Inserting it into Eq. (1), the scaling factor $b=b(t)$ is found to satisfy the Ermakov equation [26,27]

$$
\ddot{b}+\omega^{2}(t) b=\frac{\omega_{0}^{2}}{b^{3}} .
$$

$\omega_{0}$ is in principle an arbitrary constant, which we fix as the initial frequency $\omega_{0}=\omega(0)$. The eigenfunctions of $I(t)$ are

$$
\begin{aligned}
\left\langle q \mid \phi_{n}(t)\right\rangle= & \frac{1}{\left(2^{n} n ! b\right)^{1 / 2}} \exp \left[i \frac{m}{2 \hbar}\left(\frac{\dot{b}}{b}+\frac{i \omega_{0}}{b^{2}}\right) q^{2}\right] \\
& \times H_{n}\left(\left(\frac{m \omega_{0}}{\hbar}\right)^{1 / 2} \frac{q}{b}\right),
\end{aligned}
$$

and since $I(t)$ has the structure of a generalized harmonic oscillator, i.e., quadratic but with "cross" momentum-position terms, the eigenvalues are $\lambda_{n}=(n+1 / 2) \hbar \omega_{0}$.

As a consistency check, using the relations between $\hat{q}, \hat{p}, a_{t}$, and $a_{t}^{\dagger}$ (see the Appendix), and substituting the wave function $\left\langle q \mid \phi_{n}(t)\right\rangle$ into Eq. (10), the Hamiltonian may be written as

$$
H(t)=F(t)+\frac{\dot{b}}{2 b}(\hat{p} \hat{q}+\hat{q} \hat{p})-\frac{m}{2 b^{2}}\left(\dot{b}^{2}+b \ddot{b}\right) \hat{q}^{2} .
$$

When $\omega(t)$ and, thus, $H(t)$ are given, as we assume now, the invariant eigenfunctions $\left\langle q \mid \phi_{n}(t)\right\rangle$, the $\alpha_{n}(t)$, and $F(t)$ are not free functions. In particular, $\alpha_{n}(t)$ takes, from Eq. (5), the form

$$
\alpha_{n}(t)=-\left(n+\frac{1}{2}\right) \omega_{0} \int_{0}^{t} \frac{1}{b^{2}} d t^{\prime} .
$$

This gives $\dot{\alpha}_{n}=-\left(n+\frac{1}{2}\right) \omega_{0} / b^{2}$ and $F(t)=I(t) / b^{2}$. For consistency with Eq. (21), the cross terms in Eq. (26) must cancel. This is indeed the case. If $F(t)=I(t) / b^{2}$, the Hamiltonian $H(t)$ can be finally written as

$$
H(t)=\frac{1}{2 m} \hat{p}^{2}+\frac{1}{2} m\left(\frac{\omega_{0}^{2}}{b^{4}}-\frac{\ddot{b}}{b}\right) \hat{q}^{2},
$$

which is nothing but the Hamiltonian Eq. (21) after substituting $\omega(t)$ using the Ermakov equation [Eq. (24)].

\section{B. Invariant-based engineering approach}

In the inverse-engineering approach based on LewisRiesefeld invariant theory as presented in [9], the main goal is to find a "trajectory" for the external parameter $\omega(t)$ so that the populations of the final oscillator levels are the same 
as the ones of the initial oscillator. The form of $H(t)$ as an ordinary time-dependent harmonic oscillator is therefore imposed from the start although we do not know $\omega(t)$ yet. The assumed structure of the Hamiltonian implies an invariant like Eq. (23) and phases like Eq. (27). Designing $I(t)$ first here means to design $b(t)$. Let us assume an expansion of the time-dependent harmonic oscillator with initial and final frequencies $\omega(0)=\omega_{0}$ and $\omega\left(t_{f}\right)=\omega_{f}$, respectively. To make $I(t)$ and $H(t)$ commute at $t=0$ and $t_{f}$ and have common eigenfunctions, we impose the boundary conditions

$$
\begin{gathered}
b(0)=1, \quad \dot{b}(0)=0, \quad \ddot{b}(0)=0, \\
b\left(t_{f}\right)=\sqrt{\omega_{0} / \omega_{f}}, \quad \dot{b}\left(t_{f}\right)=0, \quad \ddot{b}\left(t_{f}\right)=0,
\end{gathered}
$$

by comparing Eqs. (22) and (25) and using Eq. (24). A simple polynomial ansatz can be used to interpolate $b(t)$ at intermediate times [9]. Once $b(t)$ and, therefore, the invariant are set, the time-dependent frequency $\omega(t)$ and the shortcut Hamiltonian follow from the Ermakov equation [Eq. (24)]. This method, including the effect of gravity, has been realized experimentally [24,25], extended to Bose-Einstein condensates $[8,25]$ and also to design transport protocols $[17,18]$, and used in other applications, such as the cooling of mechanical resonators [19] or the microscopy of quantum correlations in many-body systems [20]. Bounds for the transient energies involved and the implications in refrigeration cycles were studied in [5].

\section{Invariant-based method in transitionless tracking algorithm language}

As pointed out in Sec. IIC, the invariant based method can be restated in the language of the transitionless tracking algorithm. To find the reference Hamiltonian $H_{0}(t)$ implicit in Sec. III B, we interpret the $\left|\phi_{n}(t)\right\rangle$ as the eigenstates $\left|n_{0}(t)\right\rangle$ of $H_{0}$, take $\xi_{n}(t)=\alpha_{n}(t)$, and set the eigenvalues according to Eq. (19). This gives

$$
H_{0}(t)=\left(\frac{1}{b^{2}}+\frac{\dot{b}^{2}-\ddot{b} b}{2 \omega_{0}^{2}}\right) I(t)
$$

which is a generalized harmonic oscillator. The corresponding Hamiltonian $H_{1}(t)$ takes the form

$$
H_{1}(t)=\frac{\dot{b}}{2 b}(\hat{p} \hat{q}+\hat{q} \hat{p})-\frac{m}{2 b^{2}}\left(\ddot{b} b+\dot{b}^{2}\right) \hat{q}^{2}-\left(\frac{\dot{b}^{2}-\ddot{b} b}{2 \omega_{0}^{2}}\right) I .
$$

Because of the boundary conditions [Eq. (29)], $H_{1}(t)$ vanishes at $t=0$ and $t=t_{f}$. The cross terms are canceled out in the full Hamiltonian $H(t)=H_{0}(t)+H_{1}(t)$ given by Eq. (28).

\section{Transitionless tracking algorithm (standard application)}

Unlike the previous subsection, in a more standard application of the transitionless tracking algorithm to the harmonic oscillator [15], $H_{0}(t)$ is set first as an ordinary harmonic oscillator with given frequency $\omega(t)$, i.e., as in Eq. (21), so that $|n(t)\rangle$ in Eq. (22) should be now reinterpreted as $\left|n_{0}(t)\right\rangle$.
$H_{1}(t)$ is calculated from Eq. (23), and the resulting shortcut Hamiltonian becomes

$$
H(t)=\underbrace{\frac{\hat{p}^{2}}{2 m}+\frac{1}{2} m \omega^{2}(t) \hat{q}^{2}}_{H_{0}(t)}-\underbrace{-\frac{\dot{\omega}}{4 \omega(t)}(\hat{p} \hat{q}+\hat{q} \hat{p})}_{H_{1}(t)},
$$

a generalized harmonic oscillator with cross terms that imply a nonlocal interaction [28].

\section{E. Relation to invariants}

To reinterpret the previous subsection in terms of invariants, we construct $I(t)=\sum_{n}\left|n_{0}(t)\right\rangle \lambda_{n}\left\langle n_{0}(t)\right|$, with $\lambda_{n}=E_{n}^{(0)}(0)=$ $(n+1 / 2) \hbar \omega_{0}$. Since $H_{0}(t)=\sum_{n}\left|n_{0}(t)\right\rangle E_{n}^{(0)}(t)\left\langle n_{0}(t)\right|$ with instantaneous eigenvalues $E_{n}^{(0)}(t)=(n+1 / 2) \hbar \omega(t)$, the invariant is now proportional to an ordinary harmonic oscillator

$$
I(t)=\frac{\omega_{0}}{\omega(t)} H_{0}(t)=\frac{\omega_{0}}{\omega(t)}\left[\frac{\hat{p}^{2}}{2 m}+\frac{1}{2} m \omega^{2}(t) \hat{q}^{2}\right]
$$

and $I(0)=H_{0}(0)$. Using $\dot{\xi}_{n}=-E_{n}^{(0)}(t) / \hbar+i\left\langle n_{0}(t) \mid \partial_{t} n_{0}(t)\right\rangle$, and $\left\langle n_{0}(t) \mid \partial_{t} n_{0}(t)\right\rangle=0$, then letting $\alpha_{n}(t)=\xi_{n}(t)$ and $\left|\phi_{n}(t)\right\rangle=\left|n_{0}(t)\right\rangle$, we may write down $H(t)$ from Eq. (10):

$$
H(t)=F(t)-\frac{\dot{\omega}}{4 \omega(t)}(\hat{p} \hat{q}+\hat{q} \hat{p}),
$$

but here $F(t)=H_{0}(t)=\left[\omega(t) / \omega_{0}\right] I(t)$, so we recover the Hamiltonian in Eq. (32). $I(t)$ does not commute with $H(t)$ in general. To guarantee that $I(t)$ and $H(t)$ have common eigenstates at $t=0$ and $t=t_{f}$, the boundary conditions $\dot{\omega}(0)=0$ and $\dot{\omega}\left(t_{f}\right)=0$ should be satisfied, so that $H_{1}(t)$ vanishes at the initial and final times, as discussed in Sec. II.

\section{TWO-LEVEL ATOM}

The two-level atom is another fundamental model. Speededup versions of adiabatic state preparation methods, such as rapid adiabatic passage (RAP), in a two-level atomic system may be useful in chemical reaction dynamics, laser cooling, or quantum information processing.

For the two-level atom, using $|1\rangle=\left(\begin{array}{l}0 \\ 1\end{array}\right),|2\rangle=\left(\begin{array}{l}1 \\ 0\end{array}\right)$, the time-dependent Hamiltonian which we consider, in a laser adapted interaction picture and applying the rotating wave approximation [12], is

$$
H(t)=\frac{\hbar}{2}\left(\begin{array}{lc}
\Delta & \Omega_{R} e^{i \varphi} \\
\Omega_{R} e^{-i \varphi} & -\Delta
\end{array}\right),
$$

where $\Delta=\Delta(t)$ and $\Omega_{R}=\Omega_{R}(t)$ are the time-dependent detuning and Rabi frequency, respectively, and $\varphi=\varphi(t)$ a time-dependent phase. The instantaneous eigenvectors are

$$
\begin{aligned}
& \left|n_{+}(t)\right\rangle=\cos \left(\frac{\theta}{2}\right) e^{i \varphi}|2\rangle+\sin \left(\frac{\theta}{2}\right)|1\rangle, \\
& \left|n_{-}(t)\right\rangle=\sin \left(\frac{\theta}{2}\right)|2\rangle-\cos \left(\frac{\theta}{2}\right) e^{-i \varphi}|1\rangle,
\end{aligned}
$$

with the mixing angle $\theta=\theta(t) \equiv \arccos (\Delta / \Omega)$ and eigenvalues $E_{ \pm}(t)= \pm \hbar \Omega(t) / 2$, where $\Omega(t)=\sqrt{\Delta^{2}+\Omega_{R}^{2}}$. If $\varphi=0$, and the adiabaticity condition

$$
\left|\frac{\Omega_{R} \dot{\Delta}-\dot{\Omega}_{R} \Delta}{\Omega^{3}}\right| \ll 1,
$$


is satisfied, the state evolving from $\left|\psi_{ \pm}(0)\right\rangle=\left|n_{ \pm}(0)\right\rangle$ follows the adiabatic approximation

$$
\left|\psi_{ \pm}(t)\right\rangle=\exp \left\{-\frac{i}{\hbar} \int_{0}^{t} d t^{\prime} E_{ \pm}\left(t^{\prime}\right)\right\}\left|n_{ \pm}(t)\right\rangle,
$$

whereas transitions will occur otherwise. If the state starts from $\left|n_{+}(0)\right\rangle$, the adiabatic evolution of the population of levels 1 and 2 is

$$
\begin{aligned}
& P_{1}^{\mathrm{ad}}(t)=\left|\left\langle 1 \mid n_{+}(t)\right\rangle\right|^{2}=\sin ^{2}\left(\frac{\theta}{2}\right), \\
& P_{2}^{\mathrm{ad}}(t)=\left|\left\langle 2 \mid n_{+}(t)\right\rangle\right|^{2}=\cos ^{2}\left(\frac{\theta}{2}\right) .
\end{aligned}
$$

In what follows, we shall speed up the adiabatic passage by the two methods discussed in this work.

\section{A. Invariants method}

Similarly to Sec. III B, here we shall assume beforehand a form of the Hamiltonian, in this case Eq. (35). The goal of the inverse engineering will be to design the time dependence of the functions in the assumed functional form so as to end up with the desired final populations. We parametrize first the eigenvalues and eigenstates of the invariant $I(t)$, satisfying $I(t)\left|\phi_{n}(t)\right\rangle=\lambda_{n}\left|\phi_{n}(t)\right\rangle$ consistently with orthogonality and normalization, in parallel to Eqs. (36) and (37):

$$
\begin{aligned}
& \left|\phi_{+}(t)\right\rangle=\cos \left(\frac{\gamma}{2}\right) e^{i \beta}|2\rangle+\sin \left(\frac{\gamma}{2}\right)|1\rangle, \\
& \left|\phi_{-}(t)\right\rangle=\sin \left(\frac{\gamma}{2}\right)|2\rangle-\cos \left(\frac{\gamma}{2}\right) e^{-i \beta}|1\rangle,
\end{aligned}
$$

and set $\lambda_{ \pm}= \pm \hbar \Omega_{0} / 2$. [ $\Omega_{0}$ is an arbitrary constant with units of frequency to keep $I(t)$ with dimensions of energy.] Thus, $I(t)$ can be expressed as

$$
I(t)=\frac{\hbar}{2} \Omega_{0}\left(\begin{array}{ll}
\cos \gamma & \sin \gamma e^{i \beta} \\
\sin \gamma e^{-i \beta} & -\cos \gamma
\end{array}\right),
$$

where $\beta=\beta(t)$ and $\gamma=\gamma(t)$ are auxiliary time-dependent angles. Using Eqs. (35) and (41), the Lewis-Riesenfeld phase [Eq. (5)] is now calculated as [30]

$$
\alpha_{ \pm}(t)= \pm \frac{1}{2} \int_{0}^{t}\left[\Delta\left(t^{\prime}\right)-2 \tilde{\Omega}\left(t^{\prime}\right)\right] d t^{\prime},
$$

where

$$
\tilde{\Omega}=(\Delta+\dot{\beta}) \cos ^{2}\left(\frac{\gamma}{2}\right)+\frac{\Omega_{R}}{2} \sin \gamma \cos (\beta-\varphi) .
$$

Substituting these phases into Eq. (11) and then Eq. (10), the Hamiltonian is given by

$$
H(t)=\left(\frac{2 \tilde{\Omega}-\Delta}{\Omega_{0}}\right) I(t)+i \hbar \sum_{ \pm}\left|\partial_{t} \phi_{ \pm}(t)\right\rangle\left\langle\phi_{ \pm}(t)\right|,
$$

which can be finally expressed as

$$
H(t)=\frac{\hbar}{2}\left(\begin{array}{ll}
M & N e^{i \beta} \\
N^{*} e^{-i \beta} & -M
\end{array}\right),
$$

where

$$
\begin{gathered}
M=\Delta \cos ^{2} \gamma+\Omega_{R} \sin \gamma \cos \gamma \cos (\beta-\varphi)-\dot{\beta} \sin ^{2} \gamma, \\
N=\left[\Delta \cos \gamma+\Omega_{R} \sin \gamma \cos (\beta-\varphi)+\dot{\beta} \cos \gamma\right] \sin \gamma-i \dot{\gamma} .
\end{gathered}
$$

The Hamiltonian in Eq. (45) must be equivalent to the Hamiltonian in Eq. (35); so from $M=\Delta$ and $N e^{i \beta}=\Omega_{R} e^{i \varphi}$ [30], we get the auxiliary equations

$$
\begin{gathered}
\dot{\gamma}=\Omega_{R} \sin (\beta-\varphi), \\
(\Delta+\dot{\beta}) \sin \gamma=\Omega_{R} \cos \gamma \cos (\beta-\varphi) .
\end{gathered}
$$

In general, $H(t)$ does not commute with $I(t)$ :

$$
\begin{aligned}
& {[H(t), I(t)] /\left(\hbar^{2} \Omega_{0}\right)} \\
& =\hat{\sigma}_{+}\left(\Delta \sin \gamma e^{i \beta}-\Omega_{R} \cos \gamma e^{i \varphi}\right) / 2 \\
& \quad-\hat{\sigma}_{-}\left(\Delta \sin \gamma e^{-i \beta}-\Omega_{R} \cos \gamma e^{-i \varphi}\right) / 2 \\
& \quad-\hat{\sigma}_{0} i \Omega_{R} \sin \gamma \sin (\beta-\varphi) / 2,
\end{aligned}
$$

where

$$
\hat{\sigma}_{0}=\left(\begin{array}{cc}
1 & 0 \\
0 & -1
\end{array}\right), \quad \hat{\sigma}_{+}=\left(\begin{array}{ll}
0 & 1 \\
0 & 0
\end{array}\right), \quad \hat{\sigma}_{-}=\left(\begin{array}{ll}
0 & 0 \\
1 & 0
\end{array}\right) .
$$

$[H(0), I(0)]=0$ is satisfied, if

$$
\begin{gathered}
\Delta(0) \sin \gamma(0) e^{i \beta(0)}-\Omega_{R}(0) \cos \gamma(0) e^{i \varphi(0)}=0, \\
\Delta(0) \sin \gamma(0) e^{-i \beta(0)}-\Omega_{R}(0) \cos \gamma(0) e^{-i \varphi(0)}=0, \\
\Omega_{R}(0) \sin \gamma(0) \sin [\beta(0)-\varphi(0)]=0,
\end{gathered}
$$

and there are similar equations for $t_{f}$. For population inversion, we are interested in processes starting and ending with zero $\Omega_{R}$ and some finite detuning, so we impose

$$
\Omega_{R}(0)=0, \quad \gamma(0)=v \pi,
$$

so far with arbitrary $\beta(0)$ and $\varphi(0) . v$ is an arbitrary integer. In this case, $H(0)$ and $I(0)$ have common eigenvectors, which are exactly the pure ground state $|1\rangle$ and the excited state $|2\rangle$.

Similarly, for $\left[H\left(t_{f}\right), I\left(t_{f}\right)\right]=0$,

$$
\Omega_{R}\left(t_{f}\right)=0, \quad \gamma\left(t_{f}\right)=\nu^{\prime} \pi,
$$

with arbitrary $\beta\left(t_{f}\right)$ and $\varphi\left(t_{f}\right)$, and integer $v^{\prime}$. Again, $H\left(t_{f}\right)$ and $I\left(t_{f}\right)$ share common eigenstates, which are the ground and the excited state.

Substituting the above boundary conditions into Eqs. (46) and (47), we have

$$
\dot{\gamma}(0)=0, \quad \dot{\gamma}\left(t_{f}\right)=0,
$$

whereas $\dot{\beta}(0)$ and $\dot{\beta}\left(t_{f}\right)$ will determine the value of the initial and final detunings.

We are now ready to set some ansatz for $\beta$ and $\gamma$ using appropriate boundary conditions. Once the functions $\beta$ and $\gamma$ are fixed, we can construct $\Omega_{R}$ and $\Delta$ and thus the Hamiltonian $H(t)$ with a given $\varphi$. In the following subsection, we shall provide some examples of invariant-based inverse engineering with different boundary conditions.

\section{B. Examples}

We shall apply the previous results to design fast population transfer protocols. For simplicity, we assume $\varphi=0$ and consider the Hamiltonian

$$
H(t)=\frac{\hbar}{2}\left(\begin{array}{cc}
\Delta & \Omega_{R} \\
\Omega_{R} & -\Delta
\end{array}\right),
$$


with yet unknown functions $\Omega_{R}$ and $\Delta$ that will be determined, from Eqs. (46) and (47), by

$$
\begin{gathered}
\Omega_{R}=\dot{\gamma} / \sin \beta, \\
\Delta=\Omega_{R} \cot \gamma \cos \beta-\dot{\beta} .
\end{gathered}
$$

We suppose that this Hamiltonian drives the state from $|1\rangle$ to $|2\rangle$, up to a phase factor, along the invariant eigenvector $\left|\phi_{+}(t)\right\rangle$. We set the boundary conditions $\Omega_{R}(0)=\Omega_{R}\left(t_{f}\right)=0$ and

$$
\begin{gathered}
\gamma(0)=\pi, \quad \dot{\gamma}(0)=0, \\
\gamma\left(t_{f}\right)=0, \quad \dot{\gamma}\left(t_{f}\right)=0 .
\end{gathered}
$$

As mentioned before, we have freedom to choose the values of $\beta(0)$ and $\beta\left(t_{f}\right)$. According to Eq. (57), it is useful to keep $\beta$ close to $(n+1 / 2) \pi$, so as to minimize $\Omega_{R}$ along the path, whereas the derivatives fix the initial and final detunings [see Eq. (58)], which should have opposite signs here. Moreover they should not be too large to keep $\beta$ close to the chosen reference $\beta$ value, and to avoid $\beta=0$ at some intermediate time and, thus, an infinite $\Omega_{R}$. Considering all these physical constraints, we impose

$$
\begin{gathered}
\beta(0)=-\pi / 2, \quad \dot{\beta}(0)=3 \pi /\left(2 t_{f}\right), \\
\beta\left(t_{f}\right)=-\pi / 2, \quad \dot{\beta}\left(t_{f}\right)=-3 \pi /\left(2 t_{f}\right),
\end{gathered}
$$

where the negative sign of $\beta$ [see Eq. (57) and Fig. 1(a)] keeps $\Omega_{R}$ positive, as $\dot{\gamma}$ becomes negative.

To interpolate at intermediate times, we assume a polynomial ansatz. Figure 1(a) shows $\gamma(t)=\sum_{j=0}^{3} a_{j} t^{j}$ and $\beta(t)=$ $\sum_{j=0}^{3} b_{j} t^{j}$, where the coefficients are found by solving the equations set by the boundary conditions. The time-dependent $\Omega_{R}$ and $\Delta$ calculated from Eqs. (57) and (58) are shown in Fig. 1(b). Once we have specified $H(t)$ in Eq. (56), we solve the dynamics numerically by a Runge-Kutta method with an adaptive step [see the population inversion in Fig. 1(c) for levels 1 and 2]. We have also compared the bare state populations $P_{1}$ and $P_{2}$ with the populations of the instantaneous eigenstates of $H(t), P_{1}^{\text {ad }}$, and $P_{2}^{\text {ad }}$. Their agreement shows that the designed protocol is in fact an adiabatic passage for the specified final time $t_{f}$.

We may impose additional conditions at an intermediate time, for example, to keep $\beta$ closer to $-\pi / 2$ :

$$
\begin{gathered}
\beta(0)=-\pi / 2, \quad \beta\left(t_{f}\right)=-\pi / 2, \quad \beta\left(t_{f} / 2\right)=-\pi / 2, \\
\dot{\beta}(0)=\pi /\left(2 t_{f}\right), \quad \dot{\beta}\left(t_{f}\right)=-\pi /\left(2 t_{f}\right),
\end{gathered}
$$

where we have also diminished the detuning. This new set of conditions requires a higher order polynomial, $\beta(t)=$ $\sum_{j=0}^{4} b_{j} t^{j}$. Figure 2 shows the results, to be compared with those of Fig. 1. Note that in Fig. 2(b), Rabi frequency and detunings are smaller than in Fig. 1(b), so smaller energies are involved. Now, the dynamical evolution is not adiabatic [see Fig. 1(c)]. The method can be further complemented by optimizing the trajectory with respect to different physical
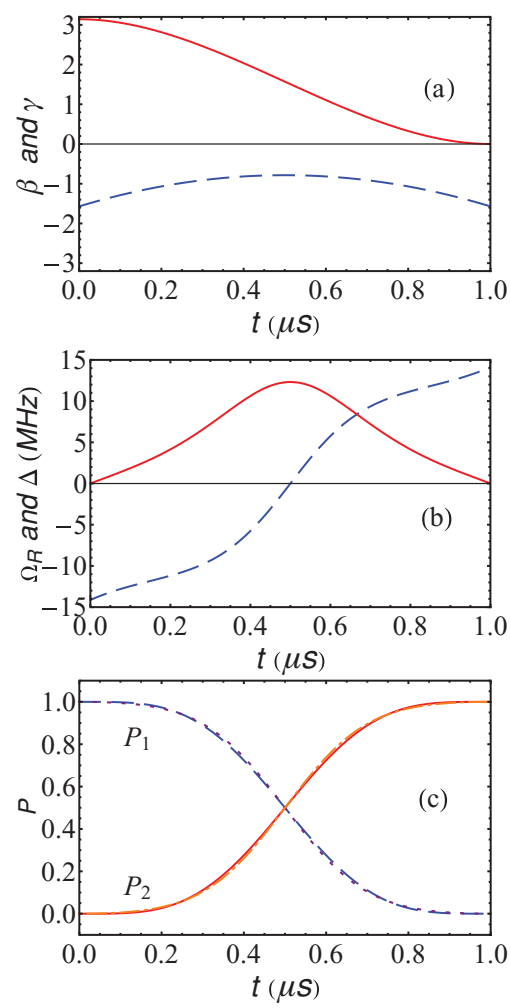

FIG. 1. (Color online) (a) Examples of polynomial ansatzes for $\gamma(t)=\sum_{j=0}^{3} a_{j} t^{j} \quad$ (solid red line) and $\beta(t)=\sum_{j=0}^{3} b_{j} t^{j}$ (dashed blue line). (b) Corresponding functions of $\Omega_{R}$ (solid red line) and $\Delta$ (dashed blue line) are determined by Eqs. (57) and (58). (c) Time evolution of the populations of levels 1 and 2: $P_{1}$ (dashed blue line), $P_{2}$ (solid red line), and adiabatic approximations $P_{1}^{\text {ad }}$ (dotted purple line) and $P_{2}^{\text {ad }}$ (dash-dotted orange line) [see Eq. (40)], hardly distinguishable from the former. $t_{f}=1 \mu \mathrm{s}$.

cost functions or constraints [16]. This will be discussed elsewhere.

\section{Invariant-based method in transitionless tracking algorithm language}

In order to reexamine the invariant-based inverseengineering approach in the language of Berry's transitionless tracking algorithm, we take $\left|\phi_{ \pm}(t)\right\rangle$ as $\left|n_{0 \pm}(t)\right\rangle$, and let $\alpha_{ \pm}(t)=$ $\xi_{ \pm}(t)$, so that Eq. (19) gives

$$
\begin{aligned}
H_{0}(t) & =\frac{\Delta \cos \gamma+\Omega_{R} \sin \gamma \cos (\beta-\varphi)}{\Omega_{0}} I(t) \\
& =\frac{2 \tilde{\Omega}-\Delta-2 \dot{\beta} \cos ^{2}(\gamma / 2)}{\Omega_{0}} I(t),
\end{aligned}
$$

and the Hamiltonian $H_{1}(t)$ in Eq. (16) is

$$
H_{1}(t)=\frac{\hbar}{2}\left(\begin{array}{lc}
-\dot{\beta} \sin ^{2} \gamma & \left(-i \dot{\gamma}+\frac{\dot{\beta}}{2} \sin 2 \gamma\right) e^{i \beta} \\
\left(i \dot{\gamma}+\frac{\dot{\beta}}{2} \sin 2 \gamma\right) e^{-i \beta} & \dot{\beta} \sin ^{2} \gamma
\end{array}\right)
$$



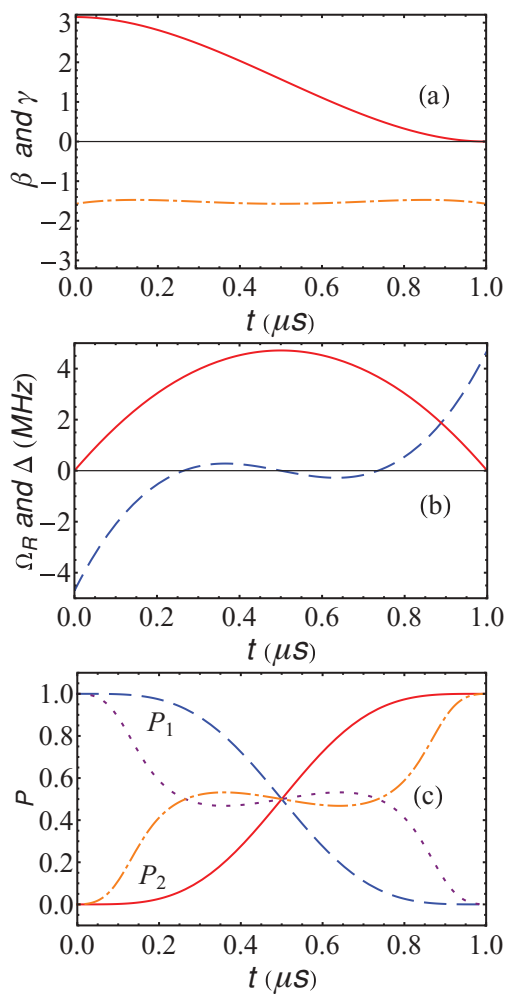

FIG. 2. (Color online) (a) Examples of polynomial ansatzes for $\gamma(t)=\sum_{j=0}^{3} a_{j} t^{j}$ (solid red line) and $\beta(t)=\sum_{j=0}^{4} b_{j} t^{j}$ (dotteddashed orange line). (b) Corresponding functions of $\Omega_{R}$ (solid red line) and $\Delta$ (dotted-dashed orange line) are determined by Eqs. (57) and (58). (c) Time evolution of the populations $P_{1}$ (dashed blue line) and $P_{2}$ (solid red line); adiabatic approximations $P_{1}^{\text {ad }}$ (dotted purple line) and $P_{2}^{\text {ad }}$ (dotted-dashed orange line) for comparison. $t_{f}=1 \mu \mathrm{s}$.

Using the boundary conditions [Eqs. (53)-(55)], $H_{1}(t)$ vanishes at $t=0$ and $t=t_{f}$.

\section{Transitionless tracking algorithm}

Let us now apply Berry's transitionless tracking algorithm taking the interaction picture Hamiltonian (35) as the reference Hamiltonian [12]:

$$
H_{0}(t)=\frac{\hbar}{2}\left(\begin{array}{lc}
\Delta & \Omega_{R} e^{i \varphi} \\
\Omega_{R} e^{-i \varphi} & -\Delta
\end{array}\right) .
$$

Choosing $\left|n_{ \pm}(t)\right\rangle$ given by Eqs. (36) and (37) as $\left|n_{0 \pm}(t)\right\rangle$, the driving Hamiltonian [Eq. (16)] becomes in this case,

$$
H_{1}(t)=\frac{\hbar}{2}\left(\begin{array}{lc}
-\dot{\varphi} \sin ^{2} \theta & \left(-i \dot{\theta}+\frac{\dot{\varphi}}{2} \sin 2 \theta\right) e^{i \varphi} \\
\left(i \dot{\theta}+\frac{\dot{\varphi}}{2} \sin 2 \theta\right) e^{-i \varphi} & \dot{\varphi} \sin ^{2} \theta
\end{array}\right) .
$$

For $\varphi=0$, this reduces to

$$
H_{1}(t)=\frac{\hbar}{2}\left(\begin{array}{lc}
0 & -i \Omega_{a} \\
i \Omega_{a} & 0
\end{array}\right)
$$

where $\Omega_{a} \equiv \dot{\theta}=\left(\dot{\Omega}_{R} \Delta-\Omega_{R} \dot{\Delta}\right) / \Omega^{2}$. In [12], this was used to speed up an Allen-Eberly scheme for $H_{0}(t)$ and achieve fast population transfer. To determine the physical meaning and realizability of the results, we may to go back to the original Schrödinger picture as discussed in [12]. We summarize here the conclusions for completeness: implementing $H(t)=H_{0}(t)+H_{1}(t)$ implies using two lasers with the same frequency, orthogonal polarization, and time-dependent intensities but different intensity shapes [12]. An alternative is to drive the system with $H_{1}(t)$ only, without $H_{0}(t)$. In the Schrödinger picture, this amounts to act with one laser and to perform level shift engineering to modulate the transition frequency so as to leave $\Delta=0$ in the interaction picture [12]. Note that these complications (an extra laser or the need for level-shift engineering) do not arise from the results of the previous subsection.

\section{E. Relation to invariants}

Let us now reinterpret the previous (standard) Berry's transitionless tracking algorithm in terms of invariant theory. In the language of Lewis-Riesefeld invariant theory, we can construct an invariant as $I(t)=\sum_{ \pm}\left|n_{0 \pm}(t)\right\rangle \lambda_{ \pm}\left\langle n_{0 \pm}(t)\right|$, where $\lambda_{ \pm}= \pm \hbar \Omega_{0} / 2$, with matrix form

$$
I(t)=\frac{\hbar}{2} \Omega_{0}\left(\begin{array}{ll}
\cos \theta & \sin \theta e^{i \varphi} \\
\sin \theta e^{-i \varphi} & -\cos \theta
\end{array}\right) .
$$

With the choice of $\Omega_{0}=\Omega(0)$, then $\lambda_{ \pm}=E_{ \pm}^{(0)}(0)$ and $I(0)=H_{0}(0)$. Since $H_{0}(t)=\sum_{ \pm}\left|n_{0 \pm}(t)\right\rangle E_{ \pm}^{(0)}(t)\left\langle n_{0 \pm}(t)\right|$ with instantaneous eigenvalues $E_{ \pm}^{(0)}(t)= \pm \hbar \Omega(t) / 2$, we have $H_{0}(t)=\left[\Omega(t) / \Omega_{0}\right] I(t)$. Using $\quad \dot{\xi}_{ \pm}=-E_{ \pm}^{(0)}(t) / \hbar+$ $i\left\langle n_{0 \pm}(t) \mid \partial_{t} n_{0 \pm}(t)\right\rangle$ and $\left\langle n_{0 \pm}(t) \mid \partial_{t} n_{0 \pm}(t)\right\rangle= \pm i \dot{\varphi} \cos ^{2}(\theta / 2)$, then letting $\left|\phi_{ \pm}(t)\right\rangle=\left|n_{0 \pm}(t)\right\rangle$ and $\alpha_{ \pm}(t)=\xi_{ \pm}(t)$, we may write $H(t)$ from Eq. (10). Canceling terms, this gives exactly the Hamiltonian $H_{0}(t)+H_{1}(t)$ in the previous subsection. $I(t)$ does not commute with $H(t)$ in general, but, when the boundary conditions

$$
\begin{gathered}
\theta(0)=v \pi, \quad \dot{\theta}(0)=0, \\
\theta\left(t_{f}\right)=v^{\prime} \pi, \quad \dot{\theta}\left(t_{f}\right)=0,
\end{gathered}
$$

are satisfied, $H_{1}(t)$ will vanish at initial and final times.

\section{CONCLUSION}

In previous publications, we applied and compared two methods to speed up adiabatic processes through nonadiabatic shortcuts: Berry's transitionless tracking algorithm and the invariant-based inverse-engineering approach. Their differences were emphasized, in particular, in time-dependent harmonic oscillators [15] or the transport of particles by a moving trap [17]. The message here is quite different, even opposite: we point out now that in fact both approaches share a common ground of concepts and structure. There is, however, no contradiction. It is indeed possible to interpret a particular inverse-engineering operation using either the language of transitionless tracking or invariants approaches, and consider them to be potentially equivalent. The explanation of the differences found is the large freedom to design different Hamiltonians for a given speedup goal. In other words, the different results are not fundamental but due to the particular choices that have been made to resolve that freedom in specific implementations. The choice 
of method from this point becomes thus, in part, a matter of taste, but there are also elements that make one or the other approach more natural or convenient. For example, systems with Hamiltonians that admit known structures for the invariants are easy to approach with the invariant-based method. This includes transport, expansions, rotations [27], or, as shown here, discrete level systems. The tracking algorithm can be applied in many systems where the invariants are unknown. In summary, this work provides a deeper understanding of shortcut-to-adiabaticity methods that will help to choose the most adequate approach in atomic transport, quantum gates, and generally atomic manipulation and control applications.

\section{ACKNOWLEDGMENTS}

We thank M. Berry for commenting on the manuscript. This work was supported by the Basque Government (Grant No. IT 472-10), Ministerio de Ciencia e Innovación (Grant No. FIS2009-12773-C02-01), Juan de la Cierva Programme, the National Natural Science Foundation of China (Grant No. 60806041), and the Shanghai Leading Academic Discipline Program (Grant No. S30105). E.T. acknowledges support from the Basque Government (Grant No. BFI08.151).

\section{APPENDIX: RELATIONS BETWEEN $\hat{q}, \hat{p}, a_{t}$, AND $a_{t}^{\dagger}$}

We can rewrite the dynamical invariant $I(t)$ in Eq. (23) as

$$
I(t)=\frac{1}{2}\left[\frac{m \omega_{0}^{2}}{b^{2}} \hat{q}^{2}+\frac{1}{m} \hat{\pi}^{2}\right]=\hbar \omega_{0}\left(a_{t}^{\dagger} a_{t}+\frac{1}{2}\right),
$$

in terms of the time-dependent creation and annihilation operators $a_{t}$ and $a_{t}^{\dagger}$, which are defined by the following relations

$$
\begin{aligned}
& a_{t}=\sqrt{\frac{m \omega_{0}}{2 \hbar}}\left[\frac{1}{b} \hat{q}+\frac{i}{m \omega_{0}}(b \hat{p}-m \dot{b} \hat{q})\right], \\
& a_{t}^{\dagger}=\sqrt{\frac{m \omega_{0}}{2 \hbar}}\left[\frac{1}{b} \hat{q}-\frac{i}{m \omega_{0}}(b \hat{p}-m \dot{b} \hat{q})\right],
\end{aligned}
$$

and satisfy the canonical commutation relation $\left[a_{t}, a_{t}^{\dagger}\right]=1$. Thus, the operators $\hat{q}$ and $\hat{p}$ can be expressed by

$$
\begin{gathered}
\hat{q}=\frac{b}{2} \sqrt{\frac{2 \hbar}{m \omega_{0}}}\left(a_{t}+a_{t}^{\dagger}\right), \\
\hat{p}=\frac{i}{b} \sqrt{\frac{\hbar m \omega_{0}}{2}}\left(a_{t}^{\dagger}-a_{t}\right)+\dot{b} \sqrt{\frac{\hbar m}{2 \omega_{0}}}\left(a_{t}+a_{t}^{\dagger}\right) .
\end{gathered}
$$

These relations simplify the calculation of $i \hbar \sum_{n}\left|\partial_{t} \phi_{n}(t)\right\rangle\left\langle\phi_{n}(t)\right|$, as in [15].
[1] M. A. Rowe, A. Ben-Kish, B. DeMarco, D. Leibfried, V. Meyer, J. Beall, J. Britton, J. Hughes, W. M. Itano, B. Jelenkovic, C. Langer, T. Rosenband, and D. J. Wineland, Quant. Inf. Comp. 2, 257 (2002).

[2] A. E. Leanhardt, T. A. Pasquini, M. Saba, A. Schirotzek, Y. Shin, D. Kielpinski, D. E. Pritchard, and W. Ketterle, Science 301, 1513 (2003).

[3] P. Salamon, K. H. Hoffmann, Y. Rezek, and R. Kosloff, Phys. Chem. Chem. Phys. 11, 1027 (2009).

[4] Y. Rezek, P. Salamon, K. H. Hoffmann, and R. Kosloff, Europhys. Lett. 85, 30008 (2009).

[5] X. Chen and J. G. Muga, Phys. Rev. A 82, 053403 (2010).

[6] R. Reichle, D. Leibfried, R. B. Blakestad, J. Britton, J. D. Jost, E. Knill, C. Langer, R. Ozeri, S. Seidelin, and D. J. Wineland, Fortschr. Phys. 54, 666 (2006).

[7] S. Masuda and K. Nakamura, Phys. Rev. A 78, 062108 (2008).

[8] J. G. Muga, X. Chen, A. Ruschhaupt, and D. Guéry-Odelin, J. Phys. B 42, 241001 (2009).

[9] X. Chen, A. Ruschhaupt, S. Schmidt, A. del Campo, D. GuéryOdelin, and J. G. Muga, Phys. Rev. Lett. 104, 063002 (2010).

[10] M. V. Berry, J. Phys. A 42, 365303 (2009).

[11] M. Murphy, L. Jiang, N. Khaneja, and T. Calarco, Phys. Rev. A 79, 020301(R) (2009).

[12] X. Chen, I. Lizuain, A. Ruschhaupt, D. Guéry-Odelin, and J. G. Muga, Phys. Rev. Lett. 105, 123003 (2010).

[13] S. Masuda and K. Nakamura, Proc. R. Soc. A 466, 1135 (2010).

[14] S. Masuda and K. Nakamura, e-print arXiv:1004.4108.

[15] J. G. Muga, X. Chen, S. Ibáñez, I. Lizuain, and A. Ruschhaupt, J. Phys. B 43, 085509 (2010).
[16] D. Stefanatos, J. Ruths, and J.-S. Li, Phys. Rev. A 82, 063422 (2010).

[17] E. Torrontegui, S. Ibáñez, X. Chen, A. Ruschhaupt, D. Guéry-Odelin, and J. G. Muga, Phys. Rev. A 83, 013415 (2011).

[18] E. Torrontegui, X. Chen, M. Modugno, S. Schmidt, A. Ruschhaupt, and J. G. Muga, e-print arXiv:1103.2532.

[19] Y. Li, L.-A. Wu, and Z.-D. Wang, Phys. Rev. A 83, 043804 (2011).

[20] A. del Campo, e-print arXiv:1103.0714.

[21] A. Couvert, T. Kawalec, G. Reinaudi, and D. Guéry-Odelin, Europhys. Lett. 83, 13001 (2008).

[22] J. Grond, J. Schmiedmayer, and U. Hohenester, Phys. Rev. A 79, 021603 (2009).

[23] J. Grond, G. von Winckel, J. Schmiedmayer, and U. Hohenester, Phys. Rev. A 80, 053625 (2009).

[24] J. F. Schaff, X. L. Song, P. Vignolo, and G. Labeyrie, Phys. Rev. A 82, 033430 (2010).

[25] J. F. Schaff, X. L. Song, P. Capuzzi, P. Vignolo, and G. Labeyrie, Europhys. Lett. 93, 23001 (2011).

[26] H. R. Lewis and W. B. Riesenfeld, J. Math. Phys. 10, 1458 (1969).

[27] M. A. Lohe, J. Phys. A 42, 035307 (2009).

[28] M. V. Berry and G. Klein, J. Phys. A 17, 1805 (1984).

[29] V. V. Dodonov, A. B. Klimov, and D. E. Nikonov, J. Math. Phys. 34, 3391 (1993).

[30] Y.-Z. Lai, J.-Q. Liang, H. J. W. Müller-Kirsten, and J.-G. Zhou, Phys. Rev. A 53, 3691 (1996); J. Phys. A 29, 1773 (1996). 